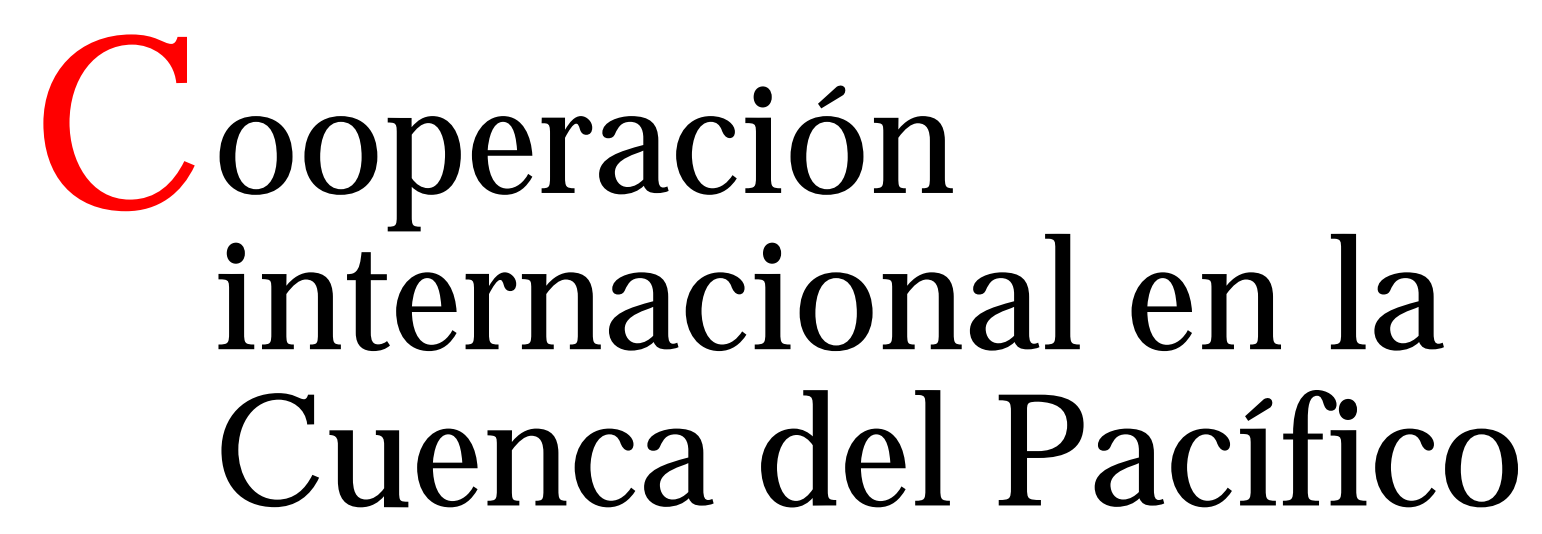

\title{
Principales factores de (in)estabilidad y la seguridad en Asia-Pacífico
}

$\mathbf{I}^{\mathrm{n}}$ ntroducción

Con el fin de la Guerra Fría y el colapso de la entonces Unión Soviética, se experimentó un cambio en el ambiente que se percibía en el contexto internacional con respecto a la confrontación, en todos los ámbitos, de los dos superpoderes: la mencionada Unión Soviética y Estados Unidos. Con esa amenaza diluida, o cuando menos disminuida sustancialmente, $\mathrm{y}$ la aparición de otras condiciones en la región de Asia-Pacífico, la atención se ha volcado hacia otros factores que pudieran trastocar la estabilidad relativa del área, lo cual, por su importancia para el resto del mundo, pudiera tener implicaciones negativas no sólo para la región sino para el orbe entero.

En el presente trabajo se abordarán los factores más importantes que tienen que ver con la preocupación por mantener la estabilidad relativa de que ha gozado la región AsiaPacífico. En primer lugar, el asunto de Taiwan $\mathrm{y}$ el posible aumento de fricciones entre China y Estados Unidos, sin dejar de lado a Japón; en segundo lugar, el tema de la península coreana, cuyo desenlace dista aún de estar claro, por lo que su devenir impactará de una u otra manera

* Investigador del Departamento de Estudios del Pacífico de la Universidad de Guadalajara.

ORCID http://orcid.org/0000-0003-0344-6895 la situación en la región, y, finalmente, las disputas cada vez más fuertes en el Mar del Sur de China, cuyas consecuencias pueden ser también imprevistas, dada la participación de varios países en el conflicto.

\section{El factor Taiwan}

El común denominador de las relaciones entre China y Estados Unidos desde el fin de la Guerra Fría ha sido el conflicto. De aquí que "los dos países participaron en confrontaciones en tres campos: derechos humanos, no proliferación [de armas] y Taiwan." ${ }^{1}$ Los primeros dos temas actualmente han sido superados y ya no constituyen puntos álgidos en la agenda, aunque sigan presentes de manera constante en la relación bilateral. De esta manera, el único campo de confrontación que sigue vigente entre China y Estados Unidos es el caso de Taiwan.

China define gran parte de su relación con Estados Unidos en función de lo que suceda en el tema de Taiwan y la presencia militar de aquél en Asia. Con ambos asuntos en particular tiene que ver la alianza entre Estados Unidos y Japón. Por un lado, China tiene la percepción de que la alianza en cuestión permite la proyección militar de Estados Unidos y Japón, las dos naciones más poderosas del mundo, a través de toda la región de Asia-Pacífico. Por el otro, China ve los nuevos 
principios del Tratado de Seguridad entre Estados Unidos y Japón como un factor significativo, cuando no una amenaza, para la unificación con Taiwan. Estos nuevos principios son vistos como elementos que pueden prolongar el statu quo, es decir la separación existente, en el mejor de los casos, y como alentadores de Taiwan hacia un mayor distanciamiento de China, en el peor. ${ }^{2}$

En cualquiera de estos escenarios, lo más preocupante para los analistas del tema es la postura inflexible de China sobre el particular, al asegurar reiteradamente que Taiwan es parte inseparable de China y que "la posición china ha sido clara y consistente y permanecerá inamovible en el futuro, sin importar el estado de la relación entre China y Estados Unidos." ${ }^{3}$ Esta postura se vio reflejada en una serie de ejercicios militares y prueba de misiles por parte del país asiático en las cercanías del estrecho de Taiwan, entre julio de 1995 y marzo de 1996. La intención de esa demostración de fuerza fue disuadir a Taiwan de sus ánimos de independencia. Sin embargo, otro de sus principales objetivos fue Estados Unidos. El mensaje para éste fue que tomaba sumamente en serio el caso de Taiwan y que estaba preparada para usar la fuerza, si era necesaria, para lograr la unión de ambas, a pesar de su intervención. $^{4}$

La posición de Estados Unidos, por otra parte, permanece de alguna manera ambigua, aunque, dada la importancia estratégica de Taiwan en la región de Asia-Pacífico y los intereses vitales de aquél en su relación con éste, es casi imposible que pase por alto cualquier cosa que pudiera comprometer el statu quo. Lo anterior no significa que Estados Unidos propicie una confrontación con China por este asunto, pero tampoco se descarta la posibilidad de una intervención militar limitada por parte del primero, con ayuda de Japón, si China iniciara alguna acción militar contra Taiwan con el fin de lograr la unificación. ${ }^{5}$ La posición ambigua de Estados Unidos, luego, es deliberada, con la intención de que ninguna de las otras dos partes involucradas estén seguras de su reacción en el caso de un conflicto armado.
Japón, por su parte, mantiene una posición más definida al asegurar que el término "áreas circundantes de Japón" incluye, o por lo menos no puede excluir, a Taiwan. Así lo han definido algunos funcionarios del gobierno japonés, principalmente Shunji Yanai, viceministro de Relaciones Exteriores en 1997, quien aseguró que la visión oficial, desde 1960, era que Taiwan se encontraba dentro de la región del "lejano oriente" definida por el tratado de seguridad entre Estados Unidos y Japón. Esto preocupa a China en el sentido de que la inclusión del estrecho de Taiwan, en los parámetros de la alianza militar entre ambos países, ha creado una excusa más para interferir en los asuntos internos de éste, aumentando así la probabilidad de una intervención militar conjunta en caso de surgimiento de alguna crisis en relación con tal asunto. ${ }^{6}$

$\mathrm{Al}$ respecto, China mantiene su postura de que éste es parte de sus asuntos internos y los intentos por excluirlo, asegura, violaría su soberanía y sería totalmente inaceptable a la luz del concepto de una sola China. Por esta razón, argumenta que Japón debería restringir la cooperación con Estados Unidos, en materia de seguridad, a la relación bilateral y no inmiscuirse en la cuestión de Taiwan, ni con cualquier cosa que pudiera dañar la soberanía de aquélla.

Otro aspecto que se tiene que tomar en cuenta en el tema de Taiwan es la dinámica interna de éste. La democratización cada vez mayor de los asuntos internos de esta isla, hace más difícil su situación con China. Ahora, el avance en las relaciones entre ambas partes y la posibilidad de dar respuesta a algunas de las demandas y expectativas de China, no sólo dependerá de los líderes políticos del primero, sino que se tendría que tomar en cuenta, al menos, al electorado en general, a la prensa, a los miembros de la legislatura y a los líderes de los demás partidos políticos. Así, cuanta mayor hostilidad se perciba de parte de éste, menores serán las posibilidades de obtener apoyo para las iniciativas de acercamiento entre las partes; $\mathrm{y}$, por el contrario, si se percibe que el 
mejoramiento de las relaciones salvaguarda sus intereses fundamentales, entonces éstas se verán aún más fortalecidas, pues será mayor su aceptación por parte de los actores principales. ${ }^{7}$

\section{El tema de la península coreana}

A pesar de que la estabilidad en la península coreana parece haber mejorado, dado el estancamiento prolongado de Corea del Norte, no cabe duda de que la situación aún se mantiene volátil y peligrosa. Gran parte de las dudas, con respecto a la situación en la zona, tuvieron que ver con una relativa incertidumbre política, la crisis económica que siguió a la muerte de Kim Ill Sung y la posibilidad incierta de lograr un aterrizaje suave para Corea del Norte, pero aún más preocupante fue la percepción del peligro que significaba la proliferación de misiles y armas nucleares, que la misma situación de crisis hacía más difícil de controlar. ${ }^{8}$

Precisamente en este aspecto reside la principal preocupación de Corea del Sur, al asegurar que la península es indudablemente el lugar del mundo más fuertemente armado, donde prevalece aún la estructura anacrónica de la Guerra Fría. Debido a esto, la seguridad en el noreste de Asia sigue haciendo frente a una gran incertidumbre, por lo que la situación imperante atrae fuertemente la atención. ${ }^{9}$

Pero no sólo Corea del Sur se preocupa por su vecino del norte, sino que también Japón lo tiene considerado como uno de los principales focos de atención. Durante los años sesenta y setenta el gobierno japonés sostuvo que la amenaza del Corea del Norte no era significativa. Sin embargo, posteriormente al lanzamiento, el 31 de agosto de 1998, del misil denominado Taepodong ${ }^{10}$ sobre Japón, éste reaccionó inmediatamente y cambió su percepción sobre la amenaza que representaba Corea del Norte para su seguridad, de modo que lo colocó entre sus amenazas más significativas. ${ }^{11}$

También Estados Unidos ve en Corea del Norte una amenaza latente para la estabilidad en la región. La preocupación fundamental es con respecto al desarrollo de la energía nuclear y de la tecnología para fabricación de misiles, lo que combinado con la posibilidad de su diseminación hacia otras regiones del mundo, hace aún más apremiante la necesidad de sofocar dicha amenaza. Esta preocupación se mantiene aun cuando se ha llegado a acuerdos que limitan la capacidad de actuación del gobierno norcoreano, pues la desconfianza imperante hacia el cumplimiento de éstos es todavía muy alta entre las partes. Así, Estados Unidos ha estado supervisando algunas instalaciones subterráneas que aparentemente podrían estarse usando para albergar equipo para procesar energía nuclear. ${ }^{12}$ Aún más, la Agencia de Energía Atómica de las Naciones Unidas realizó, a principios de este año, su primera visita oficial a un laboratorio nuclear en Corea del Norte, pues se teme que dicho sitio se haya estado utilizando para producir cantidades no especificadas de armamento nuclear. ${ }^{13}$

Por otra parte, Corea del Norte cree que la política de Estados Unidos hacia ellos es de hostilidad y, por esta razón, ha enfriado sus relaciones con Corea del Sur, que apenas en el año 2000 parecían entrar en un proceso de acercamiento y de contacto, y había aliviado las tensiones entre ambas. Esto pone también en peligro el acuerdo para garantizar el desarrollo y uso de la energía nuclear en el país, con fines pacíficos, debido a su negativa a permitir, a los inspectores de las Naciones Unidas, una revisión más detallada de las instalaciones ya mencionadas. De esta manera, "ahora ese pacto está en un punto crucial en el cual este se descarrilará, encendiendo una crisis de seguridad, o surgirá fortalecido."14

\section{El tema del Mar del Sur de China}

A pesar de que se cree que "es menos probable que China recurra al uso de la fuerza en el Mar del Sur de China que sobre Taiwan", ${ }^{15}$ la situación imperante ahí no deja de ser una de las principales amenazas para la seguridad de la región Asia-Pacífico. La participación de varios países de la zona, principalmente Brunei, China, Filipinas, Malasia, Taiwan y Vietnam, 
en la disputa por una serie de islas localizadas en sus inmediaciones, hace que el asunto se torne sumamente difícil de manejar.

La principal fuente de preocupación en el tema es China. La posición de ésta, al respecto, es que sus reclamos tienen una raíz histórica y "son irrefutables o indisputables" puesto que está en juego su soberanía. La "agresividad progresiva" mostrada por China al tratar el tema con los demás participantes en el conflicto, y su falta de disposición a tratarlo de manera multilateral, ha dejado a estos últimos con pocos recursos a los cuales recurrir para tratar de avanzar en la resolución del mismo. ${ }^{16}$

Cuando chocaron las fuerzas navales de China y Vietnam, en marzo de 1988, tres embarcaciones vietnamitas fueron hundidas y murieron más de setenta soldados. Entonces Filipinas y Malasia se alarmaron y, dejando de lado sus propias diferencias, establecieron un plan conjunto para hacer frente a China. Fue sólo hasta 1992 cuando, a través de la Asociación de Naciones del Sudeste de Asia (ASEAN, por sus siglas en inglés), se hizo una declaración para conciliar la disputa a través de negociaciones. Sin embargo, a principios de 1995 se descubrió, por parte de Filipinas, que China había iniciado la construcción de algunas instalaciones en el arrecife de Mischief, localizado dentro de las 200 millas de la zona económica exclusiva del primero. A partir de entonces, ASEAN intensificó sus esfuerzos para tratar de disciplinar a China. Así, en la reunión del Foro Regional de ASEAN (ARF, también por sus siglas en inglés) llevada a cabo en julio de 1995, se presionó a China para que cumpliera la declaración de 1992 acordada en la ASEAN. No obstante, China continuó renuente a aceptar los esfuerzos para tratar el asunto en pláticas multilaterales, a pesar de que había seguido asistiendo a las reuniones en que se abordaba el tema dentro del mismo ARF y en los talleres organizados por Indonesia sobre el problema del Mar del Sur de China, además de haber declarado que se sujetaría a lo estipulado en la Convención de las Naciones Unidas sobre la Ley de los Océanos de 1982. ${ }^{17}$

Finalmente, hacia los últimos meses de
1998 se denunció que China seguía ampliando las instalaciones en el arrecife de Mischief, a lo que Filipinas respondió tratando de llegar a un acuerdo con Malasia y Vietnam para invitar a Estados Unidos a las negociaciones entre ellos y China. La negativa por parte de esos dos países dejó sólo a Filipinas, que tampoco pudo obtener un apoyo definitivo por parte de Estados Unidos, al proponer que mejor se tratara de llegar a un arreglo pacífico en la disputa territorial. ${ }^{18}$ Aún más, ASEAN tampoco quiso comprometer demasiado su relación con China y optó por desechar la propuesta de tratar formalmente el asunto en el ARF.

Todo indica que el problema sigue latente, aunque no ha llegado a manifestarse de manera sustancial y en grandes proporciones, pero incidentes menores han seguido sucediendo en la zona del Mar del Sur de China, tales como la ocupación de algunos arrecifes de menor tamaño, la realización de ejercicios militares y el estacionamiento de tropas en las instalaciones construidas. Esto hace pensar que la situación no deja de ser amenazante, pues "todos los participantes, con la excepción de Brunei, han buscado fortalecer sus reclamos estacionando tropas en algunos de los arrecifes", ${ }^{19}$ lo que constituye un foco rojo de consecuencias impredecibles.

\section{Conclusiones}

Después de la revisión de los tres factores principales de preocupación para la seguridad en la región Asia-Pacífico, nos podemos dar cuenta de la seriedad de las amenazas a la relativa estabilidad regional que ha permitido el crecimiento y desarrollo de los países de la zona a tasas altas y constantes durante las últimas décadas, hasta antes de la crisis de 1997-1998. La necesidad de seguir gozando de esa estabilidad, fundamental para los planes de recuperación de la mayoría de las economías de la región, hace que la percepción de las posibles repercusiones de los factores aquí abordados se agudice.

Sin embargo, sería ingenuo negar que existe algún peligro en la zona del estrecho de 
Taiwan. Las posiciones, en algunos momentos irreconciliables, entre las partes, hace temer el peor de los escenarios, en el que indudablemente ninguno de los participantes saldría ganando y, por el contrario, la mayor parte de la región se vería afectada, aun cuando no se vieran involucrados directamente en la confrontación resultante.

Por otra parte, la situación en la península coreana muestra que no se descarta una posible confrontación militar. $Y$ aun cuando elimináramos tal posibilidad, lo que está muy lejos de contemplarse sin lugar a dudas, quedaría por ver el manejo y el resultado de la resolución pacífica del conflicto. Con seguridad, también, una actitud moderada por parte de Corea del Norte plantearía bastantes retos y las implicaciones se dejarían sentir no sólo en la parte correspondiente a la península, sino en la mayoría de los países de la región, sobre todo los que comparten sus fronteras y los localizados en las inmediaciones.

Por último, los problemas en el Mar del Sur de China indican que la continua falta de avance significativo en la resolución del conflicto, y su permanente posposición, puede estar sólo acumulando tensiones que pueden llevar a un desenlace inesperado. Tal escenario es indeseable porque fácilmente puede involucrar a gran número de países en una posible confrontación, puesto que son varios los participantes en las disputas territoriales de la zona, y afectaría muchos intereses, tomando en cuenta la importancia estratégica del Mar del Sur de China para la región de Asia-Pacífico.

\section{Notas}

1 Chu Shulong, China and the U.S.-Japan and U.S.Korea Alliances in a Changing Northeast Asia, Project Discussion Papers del Asia/Pacific Research Center, Institute for International Studies, Stanford University, junio de 1999, p. 15. Los documentos de esta serie están disponibles en versión electrónica en http:// www.stanford.edu/group/APARC.

2 Yu Bin, Containment by Stealth: Chinese Views of and Policies Toward America's Alliances with Japan and Korea after the Cold War, Project Discussion Papers del Asia/Pacific Research Center, Institute for International Studies, Stanford University, septiembre de 1999 , p. 9

3 Chu Shulong, op. cit., p. 16.

4 Andrew Scobell, Show of Force: The PLA and the 19951996 Taiwan Strait Crisis, Project Discussion Papers del Asia/Pacific Research Center, Institute for International Studies, Stanford University, enero de 1999, pp. 5-6.

5 Yu Bin, op. cit., p. 10.

6 Jianwei Wang y Xinbo Wu, Against Us or with Us? The Chinese Perspective of America's Alliances with Japan and Korea, Project Discussion Papers del Asia/Pacific Research Center, Institute for International Studies, Stanford University, mayo de 1998, p. 32.

7 Richard Bush, Political Change in Taiwan:Implications for American Policy, Project Discussion Papers del Asia/ Pacific Research Center, Institute for International Studies, Stanford University, octubre de 2000, p. 16. También véase David Lague, "Goodbye to The Mainland”, Far Eastern Economic Review, febrero 7 de 2002, pp. 33-35.

8 Chin Kin Wah y Pang Eng Fong, Relating the U.S-Korea and U.S.-Japan Alliances to Emerging Asia Pacific Multilateral Processes: An ASEAN Perspective, Project Discussion Papers del Asia/Pacific Research Center, Institute for International Studies, Stanford University, marzo de 2000, pp. 9-10.

9 ASEAN Regional Forum, Annual Security Outlook 2000, p. 25. Disponible en versión electrónica en: http:/ /www.aseansec.org./amm/aso/. Véase también Chung Min Lee, "North Korean Missiles: Strategic Implications and Policy Responses", The Pacific Review, vol. 14, núm. 1, 2001, pp. 85-120.

10 El 31de agosto de 1998 Corea del Norte lanzó este misil sobre el espacio aéreo de Japón y finalmente cayó en el Océano Pacífico, lo que demostró la capacidad real de Corea del Norte de llevar a cabo un ataque directo sobre Japón.

11 Michael J. Green, Japan-ROK Security Relations: An American Perspective, Project Discussion Papers del Asia/Pacific Research Center, Institute for International Studies, Stanford University, marzo de 1999 , p. 19.

12 ASEAN Regional Forum, op. cit., p. 43.

13 Far Eastern Economic Review, "Regional Briefing", enero 24 de 2002, p. 11. Véase también Far Eastern Economic Review, "Tougher Tone on Korea Checks", marzo 21 de 2002 , p. 10.

14 John Larkin y Murray Hiebert, "Meltdown", Far Eastern Economic Review, abril 4 de 2002, p. 18. El subrayado es mío.

15 Alan Collins, The Security Dilemmas of Southeast Asia, Institute of Southeast Asian Studies (ISEAS), Singapore, 2000, p. 165.

16 Ibid, p. 144. Véase también Ian James Storey, "Creeping Assertiveness: China the Philippines and the South China Sea Dispute", Contemporary Southeast Asia, vol. 21, núm. 1, abril de 1999.

17 Kristen Nordhaug, "Explaining Taiwan's Policies in the South China Sea, 1988-99", The Pacific Review, vol. 14, núm. 4, 2001, pp. 494-495. También véase Ian James Storey, op. cit., p. 99. 


\section{Cooperación internacional en la Cuenca del Pacífico}

18 Joseph Y. S. Cheng, "China's ASEAN Policy in the 1990s: Pushing for Regional Multipolarity", Contemporary Southeast Asia, vol. 21, núm. 2, agosto de 1999, pp. 190-191.

19 Alan Collins, op. cit, p. 144. T:? 